\title{
Pursuit-Evasion in Models of Complex Networks
}

\author{
Anthony Bonato, Paweł Prałat, and Changping Wang
}

Abstract. Pursuit-evasion games, such as the game of Cops and Robbers, are a simplified model for network security. In this game, cops try to capture a robber loose on the vertices of the network. The minimum number of cops required to win on a graph $G$ is its number. We present asymptotic results for the game of Cops and Robbers played in various stochastic network models, such as in $G(n, p)$ with nonconstant $p$ and in random power-law graphs. We find bounds for the cop number of $G(n, p)$ for a large range $p$ as a function of $n$. We prove that the cop number of random power-law graphs with $n$ vertices is asymptotically almost surely $\Theta(n)$. The cop number of the core of random power-law graphs is investigated, and it is proved to be of smaller order than the order of the core.

\section{Introduction}

Suppose that an intruder is loose on the vertices of a network and travels between adjacent vertices. The intruder could represent a virus, a hacker, or some other malicious agent intent on avoiding capture. A set of searchers are attempting to capture the intruder. Although placing a searcher on each vertex guarantees the capture of the intruder, it is a more interesting (and more difficult) problem to find the minimum number of searchers required to capture the intruder. A motivation for minimizing the number of searchers comes from the fact that fewer searchers require fewer resources. Networks that require a smaller number of searchers may be viewed as more secure than those that need many searchers.

(C) A K Peters, Ltd. 
A pursuit-evasion game, such as Cops and Robbers, may be viewed as a simplified model for such network security problems. The game of Cops and Robbers, introduced independently by Nowakowski and Winkler [Nowakowski and Winkler 83] and Quilliot [Quilliot 78] over twenty years ago, is played on a fixed graph $G$ and is the focus of this study. We will always assume that $G$ is undirected, simple, and finite. There are two players, a set of $k$ cops (or searchers), where $k>0$ is a fixed integer, and the robber. The cops begin the game by occupying a set of $k$ vertices. The robber then chooses a vertex, and the cops and robber move in alternate rounds. The players use edges to move from vertex to vertex. More than one cop is allowed to occupy a vertex, and the players may remain on their current vertex. The players know each other's current locations. The cops win and the game ends if at least one of the cops can eventually occupy the same vertex as the robber; otherwise, the robber wins. Since placing a cop on each vertex guarantees that the cops win, we may define the cop number, written $c(G)$, as the minimum number of cops needed to win on $G$. The cop number was introduced by Aigner and Fromme, who proved (among other things) that if $G$ is planar, then $c(G) \leq 3$ [Aigner and Fromme 84]. For a survey of results on vertex pursuit games such as Cops and Robbers, see [Alspach 06, Fomin and Thilikos 08, Hahn 07].

Over the last few years there has been an explosion of mathematical research related to stochastic models of real-world networks, especially for models of the World Wide Web graph. Many technological, social, and biological networks have properties similar to those present in the web, such as power-law degree distributions and the small-world property. Following [Chung and Lu 04], we refer to these networks as complex. For example, power laws have been observed in protein-protein interaction networks and social networks such as the one formed by scientific collaborators. While much of the earlier mathematical work on complex networks focused on designing models satisfying certain properties such as power-law degree distributions, new approaches are constantly emerging. For additional background on complex networks and their models, see [Bonato 08, Chung and Lu 04].

In this paper, which is the full version of [Bonato et al. 07b], we study vertex pursuit games in random graph models, including models for complex networks. While Cops and Robbers has been extensively studied in highly structured deterministic graphs such as graph products (see [Neufeld and Nowakowski 98]), our work is the first to consider such games in models of complex networks.

All asymptotics throughout are as $n \rightarrow \infty$. We say that an event in a probability space holds asymptotically almost surely (a.a.s.) if the probability that it holds tends to 1 as $n$ goes to infinity. For $p \in(0,1)$ or $p=p(n)$ tending to 
0 with $n$, define $\mathbb{L} n=\log _{\frac{1}{1-p}} n$. We denote the incomplete gamma function by $\Gamma(\cdot, \cdot)$.

We consider Erdős-Rényi $G(n, p)$ random graphs and their generalizations used to model complex networks. The random graph $G(n, p)$ consists of the probability space $(\Omega, \mathcal{F}, \mathbb{P})$, where $\Omega$ is the set of all graphs with vertex set $[n]=\{1,2, \ldots, n\}, \mathcal{F}$ is the family of all subsets of $\Omega$, and for every $G \in \Omega$

$$
\mathbb{P}(G)=p^{|E(G)|}(1-p)^{\left(\begin{array}{c}
n \\
2
\end{array}\right)-|E(G)|} .
$$

This space may be viewed as $\left(\begin{array}{l}n \\ 2\end{array}\right)$ independent coin flips, one for each pair of vertices, where the probability of success (that is, drawing an edge) is equal to $p$. Note that $p=p(n)$ can tend to zero as $n$ tends to infinity.

The cop number of $G(n, p)$ was studied in [Bonato et al. 07a], where the following result was proved.

Theorem I.I. Let $0<p<1$ be fixed. For every real $\varepsilon>0$ a.a.s. for $G \in G(n, p)$,

$$
(1-\varepsilon) \mathbb{L} n \leq c(G) \leq(1+\varepsilon) \mathbb{L} n .
$$

The problem of determining the cop number of $G(n, p)$ where $p=p(n)$ is a function of $n$ was left open in [Bonato et al. 07a]. In [Bollobás et al. 09] it was shown that the cop number of $G(n, p)$ is always bounded from above by $n^{1 / 2+o(1)}$ and this bound is achieved for sparse random graphs. More precisely, they showed that $c(G(n, p)) \leq 160000 \sqrt{n} \log n$ for $n p \geq 2.1 \log n$ and

$$
c(G(n, p)) \geq \frac{1}{(n p)^{2}} n^{\frac{1}{2} \frac{\log \log (n p)-9}{\log \log (n p)}}
$$

for $n p \rightarrow \infty$. Since if either $n p=n^{o(1)}$ or $n p=n^{1 / 2+o(1)}$, then a.a.s. $c(G(n, p))=$ $n^{1 / 2+o(1)}$, it would be natural to assume that the cop number of $G(n, p)$ is close to $\sqrt{n}$ also for $n p=n^{\alpha+o(1)}$, where $0<\alpha<1 / 2$. In [Euczak and Prałat 09] it was shown that the actual behavior of $c(G(n, p))$ is more complicated. The function $f:(0,1) \rightarrow \mathbb{R}$ is defined as

$$
f(x)=\frac{\log \mathbb{E}\left(c\left(G\left(n, n^{x-1}\right)\right)\right)}{\log n},
$$

where $\mathbb{E}(c(G(n, p)))$ denotes the expected value of the cop number for $G(n, p)$. The main result of [Euczak and Prałat 09] was that $f$ has an unexpected zigzag shape; see Figure 1.

In Section 1.1, we show that if $n p=n^{\alpha+o(1)}$, where $1 / 2<\alpha \leq 1$, then a.a.s.

$$
c(G(n, p))=\Theta(\log n / p)=n^{1-\alpha+o(1)}
$$




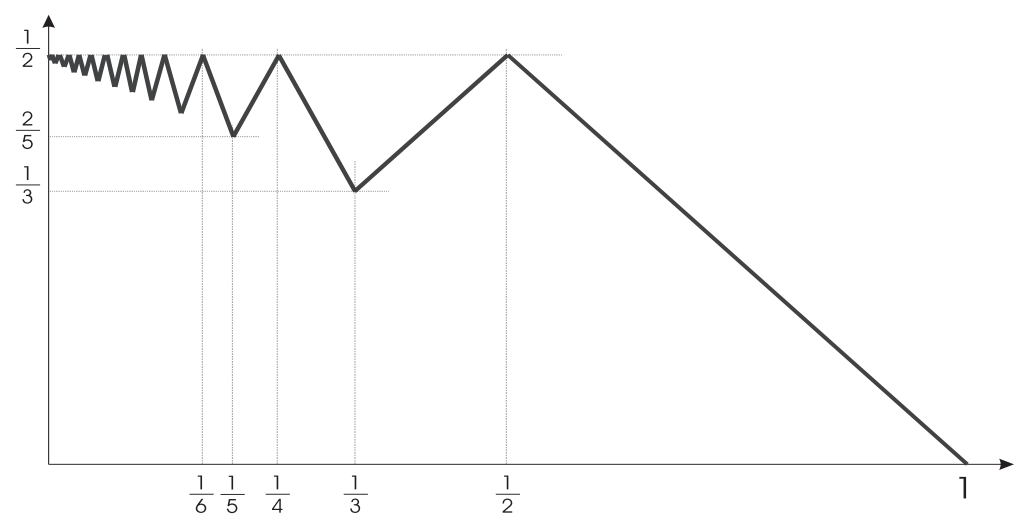

Figure I. The "zigzag" function $f$.

and $c\left(G\left(n, n^{-1 / 2+o(1)}\right)\right)=n^{1 / 2+o(1)}$ a.a.s. This result is used in [Euczak and Prałat 09], where the main focus is on $0<\alpha<1 / 2$.

Recent work of Chung and Lu supplies an extension of the $G(n, p)$ random graphs to random graphs $G(\mathbf{w})$ with given expected degree sequence w [Chung and $\mathrm{Lu}$ 03, Chung and Lu 04]. For example, if $\mathbf{w}$ follows a power-law distribution, then $G(\mathbf{w})$ supplies a model for complex networks. We determine bounds on the cop number of random power-law graphs next.

\section{I.I. Results}

We consider the cop number of classical random graphs and of random powerlaw graphs. The proofs of the results in this section may be found in Section 2. We consider the cop number of $G(n, p(n))$ when $p(n)$ is a function of $n$. We will abuse notation and refer to $p$ rather than $p(n)$. For $G(n, p)$ our main results are summarized in the following theorem.

\section{Theorem I.2.}

(a) Suppose that $p \geq p_{0}$ where $p_{0}$ is the smallest $p$ for which

$$
p^{2} / 40 \geq \frac{\log \left(\left(\log ^{2} n\right) / p\right)}{\log n}
$$

holds. Then, a.a.s. $G \in G(n, p)$ satisfies

$$
\mathbb{L} n-\mathbb{L}\left(\left(p^{-1} \mathbb{L} n\right)(\log n)\right) \leq c(G) \leq \mathbb{L} n-\mathbb{L}((\mathbb{L} n)(\log n))+2 .
$$


(b) If $(2 \log n) / \sqrt{n} \leq p=o(1)$ and $\omega(n)$ is any function tending to infinity, then a.a.s. $G \in G(n, p)$ satisfies

$$
\mathbb{L} n-\mathbb{L}\left(\left(p^{-1} \mathbb{L} n\right)(\log n)\right) \leq c(G) \leq \mathbb{L} n+\mathbb{L}(\omega(n)) .
$$

The proof may be found in Section 2.1. By Theorem 1.2, we have the following corollary.

Corollary 1.3. If $p=n^{-o(1)}$ and $p<1$, then a.a.s. $G \in G(n, p)$ satisfies

$$
c(G)=(1+o(1)) \mathbb{L} n .
$$

Indeed, from Theorem 1.2(a) it follows that if $p$ is a constant, then

$$
c(G)=\mathbb{L} n-2 \mathbb{L} \log n+\Theta(1)=(1+o(1)) \mathbb{L} n .
$$

From part (b), for $p=n^{-o(1)}$ tending to zero with $n$, the lower bound is

$$
\begin{aligned}
\mathbb{L} n-\mathbb{L}\left(\left(p^{-1} \mathbb{L} n\right)(\log n)\right) & =\mathbb{L} n-2 \mathbb{L}\left((1+o(1)) p^{-1} \log n\right) \\
& =\mathbb{L} n-2 \mathbb{L}\left(n^{o(1)}\right) \\
& =(1+o(1)) \mathbb{L} n .
\end{aligned}
$$

Note also that for $p=n^{-a(1+o(1))}(0<a<1 / 2)$ we do not have a concentration for $c(G)$ but the following bounds hold:

$$
(1+o(1))(1-2 a) \mathbb{L} n \leq c(G) \leq(1+o(1)) \mathbb{L} n .
$$

We now describe results for the cop number of random power-law graphs. Let

$$
\mathbf{w}=\left(w_{1}, \ldots, w_{n}\right)
$$

be a sequence of $n$ nonnegative real numbers. We define a random graph model, written $G(\mathbf{w})$, as follows. Typically, vertices are integers in $[n]$. Each potential edge between $i$ and $j$ is chosen independently with probability $p_{i j}=w_{i} w_{j} \rho$, where

$$
\rho=\frac{1}{\sum_{i=1}^{n} w_{i}} .
$$

We will always assume that

$$
\max _{i} w_{i}^{2}<\sum_{i=1}^{n} w_{i}
$$


which implies that $p_{i j} \in[0,1)$. The model $G(\mathbf{w})$ is referred to as random graphs with given expected degree sequence $\mathbf{w}$. Observe that $G(n, p)$ may be viewed as a special case of $G(\mathbf{w})$ by taking $\mathbf{w}$ to be equal to the constant $n$-sequence $(p n, p n, \ldots, p n)$.

Given $\beta>2, d>0$, and a function $M=M(n)=o(\sqrt{n})$ (with $M$ tending to infinity with $n$ ), we consider the random graph with given expected degrees $w_{i}>0$, where

$$
w_{i}=c i^{-\frac{1}{\beta-1}}
$$

for $i$ satisfying $i_{0} \leq i<n+i_{0}$. The term $c$ depends on $\beta$ and $d$, and $i_{0}$ depends also on $M$; namely,

$$
c=\left(\frac{\beta-2}{\beta-1}\right) d n^{\frac{1}{\beta-1}}, \quad i_{0}=n\left(\frac{d}{M}\left(\frac{\beta-2}{\beta-1}\right)\right)^{\beta-1} .
$$

It is not hard to show (see [Chung and Lu 03, Chung and Lu 04]) that a.a.s. the random graphs with the expected degrees satisfying (1.1) and (1.2) follow a power-law degree distribution with exponent $\beta$, average degree $(1+o(1)) d$, and maximum degree $(1+o(1)) M$.

Our next theorem demonstrates that the cop number of random power-law graphs is a.a.s. $\Theta(n)$ and so is of much larger order than the logarithmic cop number of $G(n, p)$ random graphs. Hence, these results suggest that in powerlaw graphs on average a large number of cops are needed to secure the network.

Theorem I.4. For a random power-law graph $G \in G(\mathbf{w})$ with exponent $\beta>2$ and average degree d, a.a.s. the following hold:

(a) If $X$ is the random variable denoting the number of isolated vertices in $G(\mathbf{w})$, then

$$
\begin{aligned}
c(G) & \geq X \\
& =(1+o(1)) n \int_{0}^{1} \exp \left(-d \frac{\beta-2}{\beta-1} x^{-1 /(\beta-1)}\right) d x \\
& =(1+o(1))(d(\beta-2))^{\beta-1}(\beta-1)^{2-\beta} n \Gamma\left(1-\beta, d \frac{\beta-2}{\beta-1}\right) .
\end{aligned}
$$

(b) For $a \in(0,1)$, define

$$
f(a)=a+\int_{a}^{1} \exp \left(-d \frac{\beta-2}{\beta-1} a^{(\beta-2) /(\beta-1)} x^{-1 /(\beta-1)}\right) d x .
$$

Then

$$
c(G) \leq(1+o(1)) n \min _{0<a<1} f(a) .
$$




\begin{tabular}{|c|c|c|}
\hline & 10 & 20 \\
\hline 2.1 & $0.1806 / 0.2940$ & $0.5112 \times 10^{-1} / 0.1265$ \\
2.7 & $0.4270 \times 10^{-2} / 0.1895$ & $0.4205 \times 10^{-4} / 0.8261 \times 10^{-1}$ \\
\hline
\end{tabular}

Table I. Upper and lower bounds for the cop number of $G(\mathbf{w})$ for various values of $d$ (top row) and $\beta$ (left column).

The proof of Theorem 1.4 may be found in Section 2.3. We note that integrals in the statement of Theorem 1.4 do not possess closed-form solutions in general. We supply in Table 1 numerical values for lower/upper bounds of the cop number of $G(\mathbf{w})$ when $d=10,20$ and $\beta=2.1,2.7$ (note that the values of $d=10$ and $\beta=2.1$ coincide with earlier experimental results found in the web graph; see, for example, the survey [Bonato 08]).

While Theorem 1.4 suggests that a large number of cops are needed to secure complex networks against intruders, by part (a) it is the abundance of isolated vertices that makes the cop number equal to $\Theta(n)$. To overcome the issue with isolated vertices, we consider restricting the movements of the cops and robber to the subgraph induced by sufficiently-high-degree vertices.

Fix $\beta \in(2,3)$. Define the core of a graph $G$, written $\widehat{G}$, as the subgraph induced by the set of vertices of degree at least $n^{1 / \log \log n}$. Random power-law graphs with $\beta \in(2,3)$ are referred to as octopus graphs in [Chung and Lu 04], since the core is dense with small diameter $O(\log \log n)$ and the overall diameter is $O(\log n)$. For $G \in G(\mathbf{w})$, since the expected degree of vertex $i$ in $G$ is

$$
w_{i}=\frac{\beta-2}{\beta-1} d n^{1 /(\beta-1)} i^{-1 /(\beta-1)},
$$

vertices with expected degree at least $n^{1 / \log \log n}$ have labels at most

$$
i_{N}=\left(\frac{\beta-2}{\beta-1} d\right)^{\beta-1} n^{1-(\beta-1) / \log \log n} .
$$

The order of the core is written N. By Chernoff's bound,

$$
N=(1+o(1)) i_{N}-i_{0}=(1+o(1)) i_{N}=\Theta\left(n^{1-(\beta-1) / \log \log n}\right),
$$

provided that $\log M \gg(\log n) / \log \log n$. Thus,

$$
n=N^{1+(\beta-1) / \log \log N+\Theta(1) / \log ^{2} \log N} .
$$

We consider the cop number of the core of random power-law graphs (so the cop and robber are restricted to movements within the core). As vertices in the core informally represent the hubs of the network, one would suspect that the 
cop number of the core is of smaller order than the core itself. This intuition is made precise by the following theorem, which provides a sublinear upper bound for the cop number of the core. The proof is deferred to Section 2.4.

Theorem 1.5. For a random power-law graph $G \in G(\mathbf{w})$ with power-law exponent $\beta \in(2,3)$, a.a.s. the cop number of the core $\widehat{G}$ of $G$ satisfies

$$
N^{(1+o(1))(3-\beta) / \log \log N} \leq c(\widehat{G}) \leq N^{1-(1+o(1))(\beta-1)(3-\beta) /(\beta-2) \log \log n} .
$$

As the asymptotic bounds in Theorem 1.5 are not tight, it is an interesting open problem to determine the asymptotic value of the cop number of the core of random power-law graphs.

\section{Proofs of Main Results}

The remainder of this paper is devoted to the proof of our three main theorems. Each subsequent subsection contains a proof of the required bounds in each theorem. In all cases, the upper bound for the cop number is provided by a corresponding bound of the domination number. In all cases except the proof of Theorem 1.4 (which estimates the number of isolated vertices), a lower bound for the cop number is derived by considering an appropriate adjacency property. A minor detour is made in Section 2.2, which gives a concentration result for the cop number in sparse $G(n, p)$ random graphs.

\section{I. Proof of Theorem 1.2}

We first prove the upper bound in Theorem 1.2(a), and we require some background on the domination number of a graph. A set of vertices $S$ is a dominating set in $G$ if each vertex not in $S$ is joined to some vertex of $S$. The domination number of $G$, written $\gamma(G)$, is the minimum cardinality of a dominating set in $G$. A straightforward observation is that

$$
c(G) \leq \gamma(G),
$$

(place a cop on each vertex of dominating set with minimum cardinality). However, if $n \geq 1$, then $c\left(P_{n}\right)=1$ (where $P_{n}$ is a path with $n$ vertices) and $\gamma\left(P_{n}\right)=\left\lceil\frac{n}{3}\right\rceil$. The bound of (2.1) while useful, is far from tight in general. Domination in models of complex networks were considered in [Cooper et al. 05].

The upper bound in Theorem 1.2(a) follows by the following result proved in [Wieland and Godbole 01]. 
Theorem 2.I. Suppose that $p \geq p_{0}(n)$, where $p_{0}$ is the smallest $p$ for which

$$
p^{2} / 40 \geq \frac{\log \left(\left(\log ^{2} n\right) / p\right)}{\log n}
$$

holds. Then, a.a.s. $G \in G(n, p)$ satisfies

$$
\lfloor\mathbb{L} n-\mathbb{L}((\mathbb{L} n)(\log n))\rfloor+1 \leq \gamma(G) \leq\lfloor\mathbb{L} n-\mathbb{L}((\mathbb{L} n)(\log n))\rfloor+2 .
$$

For Theorem 1.2(b), the proof follows by the following theorem.

Theorem 2.2. If $p=o(1)$ and $\omega(n)$ is any function tending to infinity with $n$, then a.a.s. $G \in G(n, p)$ satisfies

$$
\gamma(G) \leq\lceil\mathbb{L} n+\mathbb{L}(\omega(n))\rceil .
$$

Proof. Since $p=o(1)$ we have that

$$
\mathbb{L} n=\frac{\log n}{-\log (1-p)}=(1+o(1)) \frac{\log n}{p} .
$$

The probability that the domination number of a random graph is at most

$$
k=\lceil\mathbb{L} n+\mathbb{L}(\omega(n))\rceil
$$

is bounded from below by the probability that any fixed set of $k$ vertices is a dominating set. But, the latter probability is equal to

$$
\begin{aligned}
\left(1-(1-p)^{k}\right)^{n-k} & \geq 1-(n-k)(1-p)^{k} \\
& \geq 1-n(1-p)^{k} \\
& \geq 1-n(1-p)^{\mathbb{L} n+\mathbb{L}(\omega(n))} \\
& =1-\frac{1}{\omega(n)} \\
& =1-o(1) .
\end{aligned}
$$

For the proofs of the lower bounds in Theorem 1.2, we employ the following adjacency property. For a fixed integer $k>0$, we say that $G$ is $(1, k)$-existentially closed (or $(1, k)$-e.c.) if for each $k$-set $S$ of vertices of $G$ and vertex $u \notin S$, there is a vertex $z \notin S$ not joined to a vertex in $S$ and joined to $u$. If $G$ is $(1, k)$-e.c., then $c(G) \geq k$ (the robber may use the property to escape to a vertex not joined to any vertex occupied by a cop). 
The lower bound in Theorem 1.2 will follow once we prove the following theorem.

Theorem 2.3. If $p>(2 \log n) / \sqrt{n}$ and

$$
k=\left\lfloor\mathbb{L} n-\mathbb{L}\left(\left(p^{-1} \mathbb{L} n\right)(\log n)\right)\right\rfloor,
$$

then a.a.s. $G \in G(n, p)$ is $(1, k)$-e.c.

Note that we do not use the condition for $p$ in the proof of the theorem. The condition is introduced in order to get a nontrivial result; the value of $k$ is negative otherwise.

Proof. Assume that $p=o(1)$. Then,

$$
\begin{aligned}
k & =\mathbb{L} n-\mathbb{L}\left((1+o(1)) \frac{\log ^{2} n}{p^{2}}\right) \\
& =\mathbb{L} n-2 \mathbb{L}\left((1+o(1)) \frac{\log n}{p}\right) .
\end{aligned}
$$

Fix $S$ a $k$-subset of vertices and a vertex $u$ not in $S$. For a vertex $x \in$ $V(G) \backslash(S \cup\{u\})$, the probability that a vertex $x$ is joined to $u$ and to no vertex of $S$ is $p(1-p)^{k}$. Since edges are chosen independently, the probability that no suitable vertex can be found for this particular $S$ and $u$ is $\left(1-p(1-p)^{k}\right)^{n-k-1}$.

Let $X$ be the random variable counting the number of $S$ and $u$ for which no suitable $x$ can be found. We then have that

$$
\begin{aligned}
\mathbb{E}(X) & =\left(\begin{array}{l}
n \\
k
\end{array}\right)(n-k)\left(1-p(1-p)^{k}\right)^{n-k-1} \\
& \leq n^{k+1}\left(1-\frac{(\mathbb{L} n)(\log n)}{n}\right)^{n(1-(\mathbb{L} n) / n)} \\
& =n^{k+1} \exp (-(\mathbb{L} n)(\log n)(1-(\mathbb{L} n) / n))(1+o(1)) \\
& =n^{k+1} \exp \left(-\left(\mathbb{L} n-(\mathbb{L} n)^{2} / n\right)(\log n)(1+o(1))\right) \\
& \leq n^{k+1} \exp \left(-\left(k+\frac{2 \log \log n}{p}-\frac{2 \log ^{2} n}{p^{2} n}\right)(\log n)(1+o(1))\right) \\
& =n^{k+1} \exp \left(-\left(k+\frac{2 \log \log n}{p}\right)(\log n)(1+o(1))\right) \\
& =o(1)
\end{aligned}
$$

where the second inequality follows by (2.2). It is also easy to show that the same argument holds for $p$ a constant. The proof now follows by Markov's inequality. 


\subsection{Extreme Cases}

It is known that if $p$ tends to one pretty fast, then the maximum degree of a random graph is very large. Formally, for a fixed nonnegative integer $k$, if $n(1-p)-\log n-k \log \log n \rightarrow-\infty$, then the maximum degree is at least $n-1-k$ a.a.s. and clearly $k+1$ is an upper bound for the cop number (one cop can occupy a vertex with maximum degree). In fact,

$$
p=p(n)=1-\left(\frac{k \log n}{n}\right)^{\frac{1}{k}}
$$

is the threshold for the cop number $k+1$ (see [Prałat 09] for more details).

We next provide a concentration result for the cop number of the random graphs $G(n, p)$ when $p$ approaches zero very fast. For example, if $p=o\left(1 / n^{2}\right)$, a.a.s. $G \in G(n, p)$ is empty. In this range of $p$, a.a.s. the cop number of $G$ is $n$. We now consider the case when $p=d / n$ for constant $d \in(0,1)$. Bollobás proved the following result [Bollobás 01].

Theorem 2.4. Let $0<d<1, p=d / n$, and $X$ be the number of tree connected components of $G(n, p)$. Then, the expectation of $X$ is

$$
\mathbb{E}(X)=u(d) n+O(1),
$$

where

$$
u(d)=\frac{1}{d} \sum_{k=1}^{\infty} \frac{k^{k-2}}{k !}\left(d e^{-d}\right)^{k} .
$$

A.a.s. $G(n, p)$ satisfies

$$
|X|=(1+o(1)) u(d) n
$$

We note that $u(d) \in(0,1)$. A graph is unicyclic if it contains exactly one cycle.

Theorem 2.5. Let $0<d<1$ and $p=d / n$. Then, a.a.s. $G \in G(n, p)$ is such that every connected component is a tree or a unicyclic graph, and there are at most $\log \log n$ vertices in the unicyclic components.

Trees are cop-win graphs, while unicyclic graphs have cop number at most 2 . Each tree component requires exactly one cop, while there are at most $2 \log \log n$ many cops needed for all the unicyclic components. Hence, the number of cops on the unicyclic components becomes negligible in contrast to the number of 
cops on the tree components. Therefore, from Theorems 2.4 and 2.5 we have the following concentration result.

Corollary 2.6. Let $0<d<1, p=d / n$. Then for the graph $G \in G(n, p)$,

$$
\mathbb{E}(c(G))=u(d) n+O(\log \log n) .
$$

A.a.s. $G \in G(n, p)$ satisfies

$$
c(G)=(1+o(1)) u(d) n .
$$

\subsection{Proof of Theorem I.4}

Proof. For the lower bound, we exploit the fact that the cop number is bounded from below by the number of isolated vertices: one cop is needed per isolated vertex. In general power-law graphs, there may exist an abundance of isolated vertices, even as much as $\Theta(n)$ many. We show that this is indeed the case for random power-law graphs.

The probability that the vertex $i$ for $i_{0} \leq i<n+i_{0}$ (that is, the vertex $i$ corresponds to the weight $w_{i}$ ) is isolated is equal to

$$
\begin{aligned}
p_{i} & =\prod_{j, j \neq i}\left(1-w_{i} w_{j} \rho\right) \\
& =\prod_{j, j \neq i} \exp \left(-w_{i} w_{j} \rho(1+o(1))\right) \\
& =\exp \left(-w_{i} \rho \sum_{j, j \neq i} w_{j}(1+o(1))\right) \\
& =\exp \left(-w_{i}(1+o(1))\right)
\end{aligned}
$$

Let $X_{i}$ be an indicator random variable for the event that the vertex $i$ is isolated. Then,

$$
\mathbb{P}\left(X_{i}=1\right)=1-\mathbb{P}\left(X_{i}=0\right)=p_{i}
$$

for $i_{0} \leq i<n+i_{0}$.

Let $X$ be the number of isolated vertices in $G(\mathbf{w})$. Since $X=\sum_{i_{0} \leq i<n+i_{0}} X_{i}$, it follows from (2.3) that the expected value of $X$ is

$$
\begin{aligned}
\sum_{i_{0} \leq i<n+i_{0}} p_{i} & =(1+o(1)) n \int_{0}^{1} \exp \left(-c(x n)^{-1 /(\beta-1)}\right) d x \\
& =(1+o(1)) n \int_{0}^{1} \exp \left(-d \frac{\beta-2}{\beta-1} x^{-1 /(\beta-1)}\right) d x .
\end{aligned}
$$


A sum of independent random variables with large enough expected value is concentrated on its expected value (see, for example, [Janson et al. 00, Theorem 2.8]). Thus, the number of isolated vertices in $G(\mathbf{w})$ is a.a.s. equal to

$$
\begin{aligned}
X & =(1+o(1)) n \int_{0}^{1} \exp \left(-d \frac{\beta-2}{\beta-1} x^{-1 /(\beta-1)}\right) d x \\
& =(1+o(1))(d(\beta-2))^{\beta-1}(\beta-1)^{2-\beta} n \int_{d \frac{\beta-2}{\beta-1}}^{\infty} t^{-\beta} e^{-t} d t \\
& =(1+o(1))(d(\beta-2))^{\beta-1}(\beta-1)^{2-\beta} n \Gamma\left(1-\beta, d \frac{\beta-2}{\beta-1}\right) .
\end{aligned}
$$

Hence, Theorem 1.4(a) follows. We note that another asymptotic expression of the number of isolated vertices in random power-law graphs has been given (see [Chung and Lu 04, Theorem 5.20]).

For the proof of the upper bound in Theorem 1.4(b), we give a bound on the domination number. Fix a constant $a \in(0,1)$, and consider the set $A \subseteq V$ of the first $\lfloor a n\rfloor-i_{0}+1=(1+o(1))$ an vertices; that is, $A=\left\{i_{0}, i_{0}+1, \ldots,\lfloor\right.$ an $\left.\rfloor\right\}$. Let $B \subseteq V \backslash A$ denote the set of vertices that do not have a neighbor in $A$. Then $D=A \cup B$ is a dominating set of $G$, and we estimate the cardinality of $D$.

Consider the vertex $i$, where $a n<i<n+i_{0}$. Since $i_{0}=o(n)$, there is $b \in(0,1]$ such that $i=b n(1+o(1))$. The probability that $i$ does not have a neighbor in $A$ is equal to

$$
\begin{aligned}
q_{i} & =\prod_{j<a n+i_{0}}\left(1-w_{i} w_{j} \rho\right) \\
& =\exp \left(-(1+o(1)) w_{i} \rho \sum_{j<a n+i_{0}} w_{j}\right) \\
& =\exp \left(-(1+o(1)) c(b n)^{-1 /(\beta-1)}(d n)^{-1} n \int_{0}^{a} c(x n)^{-1 /(\beta-1)} d x\right) \\
& =\exp \left(-(1+o(1)) d\left(\frac{\beta-2}{\beta-1}\right)^{2} b^{-1 /(\beta-1)} \int_{0}^{a} x^{-1 /(\beta-1)} d x\right) \\
& =\exp \left(-(1+o(1)) d \frac{\beta-2}{\beta-1} b^{-1 /(\beta-1)} a^{(\beta-2) /(\beta-1)}\right) .
\end{aligned}
$$

Thus, using Chernoff's bound, we obtain that a.a.s.

$$
|B|=(1+o(1)) n \int_{a}^{1} \exp \left(-d \frac{\beta-2}{\beta-1} a^{(\beta-2) /(\beta-1)} x^{-1 /(\beta-1)}\right) d x,
$$


and that a.a.s.

$$
\begin{aligned}
|D| & =|A \cup B| \\
& =(1+o(1)) n\left(a+\int_{a}^{1} \exp \left(-d \frac{\beta-2}{\beta-1} a^{(\beta-2) /(\beta-1)} x^{-1 /(\beta-1)}\right) d x\right) .
\end{aligned}
$$

As this holds for every $a \in(0,1)$, the proof of part (b) follows.

\subsection{Proof of Theorem I.5}

Proof. We first consider an upper bound for $c(\widehat{G})$ by using a dominating set. The probability that there is an edge between two given vertices in the core is at least

$$
\begin{aligned}
p_{\min } & =w_{i_{N}(1+o(1))}^{2} \rho \\
& =\frac{n^{2 / \log \log n}}{d n}(1+o(1)) \\
& =\frac{N^{(3-\beta) / \log \log N+\Theta(1) / \log ^{2} \log n}}{N} \\
& =\frac{N^{(1+o(1))(3-\beta) / \log \log N}}{N} .
\end{aligned}
$$

Hence, $\widehat{G}$ contains a random graph $G\left(N, p_{\text {min }}\right)$. Thus, using Theorem 2.2 , any set of cardinality

$$
\begin{aligned}
& k_{N}=\left\lceil\log _{1 /\left(1-p_{\min }\right)} N+\log _{1 /\left(1-p_{\min }\right)} \omega(N)\right\rceil \\
& =(1+o(1)) \frac{\log N}{p_{\min }} \\
& =\frac{N \log N}{N^{(1+o(1))(3-\beta) / \log \log N}} \\
& =N \exp \left(-\frac{(1+o(1))(3-\beta)(\log N)}{\log \log N}+\log \log N\right) \\
& =N \exp \left(-\frac{(1+o(1))(3-\beta)(\log N)}{\log \log N}\right) \\
& =\frac{N}{N^{(1+o(1))(3-\beta) / \log \log N}} \\
& =o(N)
\end{aligned}
$$

is a dominating set a.a.s. (where $\omega(N)$ is any function tending to infinity with $N)$. As this holds for any set of cardinality $k_{N}$, we obtain a smaller dominating 
set by considering only vertices with the largest expected degree. Consider the subset of vertices $U=\left\{i_{0}, i_{0}+1, \ldots, k\right\}$ of first $k-i_{0}+1$ vertices, $k \gg i_{0}$. Then,

$$
\sum_{i=i_{0}}^{k} \omega_{i}=c \int_{i_{0}}^{k} i^{-1 /(\beta-1)} d i+O(1)=(1+o(1)) c \frac{\beta-1}{\beta-2} k^{(\beta-2) /(\beta-1)} .
$$

Hence, the probability that vertex $i$ does not have a neighbor in $U$ is equal to

$$
\begin{aligned}
q(i) & =\prod_{j=i_{0}}^{k}\left(1-\omega_{i} \omega_{j} \rho\right) \\
& =\exp \left(-(1+o(1)) \omega_{i} \rho \sum_{j=i_{0}}^{k} \omega_{j}\right) \\
& =\exp \left(-(1+o(1)) \frac{\beta-2}{\beta-1} d n^{(3-\beta) /(\beta-1)} i^{-1 /(\beta-1)} k^{(\beta-2) /(\beta-1)}\right) .
\end{aligned}
$$

It is straightforward to see that for all vertices $i$ in the core,

$$
\begin{aligned}
q(i) & \leq q\left(i_{N}(1+o(1))\right) \\
& =\exp \left(-(1+o(1)) n^{(2-\beta) /(\beta-1)+1 / \log \log n} k^{(\beta-2) /(\beta-1)}\right) .
\end{aligned}
$$

Therefore, in order to make the right-hand side of $(2.5)$ equal to $o\left(n^{-1}\right)$, it is enough to take

$$
\begin{aligned}
k & =n^{1-(\beta-1) /(\beta-2) \log \log n} \log ^{2(\beta-1) /(\beta-2)} n \\
& =n^{1-(1+o(1))(\beta-1) /(\beta-2) \log \log n} \\
& =N^{1-(1+o(1))(\beta-1)(3-\beta) /(\beta-2) \log \log n} .
\end{aligned}
$$

Now, the expected number of vertices that are not dominated by $U$ is $o(1)$, and the assertion follows from Markov's inequality. The upper bound now follows.

For the lower bound, we can show that the core is a.a.s. $(1, k)-e . c$. for

$$
k=\log N / \log \log N
$$

We do not present this argument here but instead prove a better bound by considering a weaker adjacency property. We will show that a.a.s. for all subsets $S$ of vertices of cardinality

$$
K=N^{(3-\beta) / \log \log N-1 / \log ^{3 / 2} \log N}=N^{(1+o(1))(3-\beta) / \log \log N}
$$

and all vertices $u \notin S$ with $N / 3<u<2 N / 3$, there is a vertex $x \notin(S \cup\{u\})$ with $N / 3<x<2 N / 3$, not joined to a vertex in $S$ and joined to $u$. This will 
yield a lower bound of $K$ for the cop number since the robber can move only on vertices with labels between $N / 3$ and $2 N / 3$ to avoid being captured.

Note that the probability that there is an edge between a vertex in the core and a vertex with label between $N / 3$ and $2 N / 3$ is at most

$$
\begin{aligned}
p_{\max } & =M \Theta\left(n^{1 / \log \log n}\right) \rho \\
& \leq n^{-1 / 2+1 / \log \log n} \\
& =N^{-1 / 2+(3-\beta) / 2 \log \log N+\Theta(1) / \log ^{2} \log N}
\end{aligned}
$$

(using (1.3) and the fact that $M=o(\sqrt{n})$ ). A lower bound for the edge probability is given by (2.4).

Let $X$ be the random variable counting the number of ordered pairs of $K$-sets $S$ and vertices $u$ with $N / 3<u<2 N / 3$ for which no suitable vertex $x$ with $N / 3<x<2 N / 3$ can be found. Then,

$$
\begin{aligned}
\mathbb{E}(X) \leq & N^{K+1}\left(1-p_{\min }\left(1-p_{\max }\right)^{K}\right)^{\Theta(N)-K-1} \\
= & N^{K+1}\left(1-N^{-1+(3-\beta) / \log \log N+\Theta(1) / \log ^{2} \log N}\right. \\
& \left.\quad \times\left(1-N^{-1 / 2+\Theta(1) / \log \log N}\right)^{K}\right)^{\Theta(N)} \\
= & N^{K+1}\left(1-N^{-1+(3-\beta) / \log \log N+\Theta(1) / \log ^{2} \log N}\right)^{\Theta(N)} \\
= & \exp \left((K+1) \log N-N^{(3-\beta) / \log \log N+\Theta(1) / \log ^{2} \log N}\right) \\
= & \exp \left(N^{(3-\beta) / \log \log N-\Theta(1) / \log ^{3 / 2} \log N}\right) \\
& \left.\quad-N^{(3-\beta) / \log \log N+\Theta(1) / \log ^{2} \log N}\right) \\
= & \exp \left(-N^{(1+o(1))(3-\beta) / \log \log N}\right) \\
= & o(1) .
\end{aligned}
$$

The proof now follows by Markov's inequality.

\section{References}

[Aigner and Fromme 84] M. Aigner and M. Fromme. "A Game of Cops and Robbers." Discrete Applied Mathematics 8 (1984), 1-12.

[Alspach 06] B. Alspach. "Sweeping and Searching in Graphs: A Brief Survey." Matematiche 59 (2006), 5-37.

[Bonato 08] A. Bonato. A Course on the Web Graph, Graduate Studies Series in Mathematics. Providence, RI: American Mathematical Society, 2008. 
[Bollobás 01] B. Bollobás. Random Graphs. Cambridge, UK: Cambridge University Press, 2001.

[Bollobás et al. 09] B. Bollobás, G. Kun, and I. Leader. "Cops and Robbers in a Random Graph." Preprint, 2009.

[Bonato et al. 07a] A. Bonato, G. Hahn, and C. Wang. "The Cop Density of a Graph." Contributions to Discrete Mathematics 2 (2007), 133-144.

[Bonato et al. 07b] A. Bonato, P. Prałat, and C. Wang. "Vertex Pursuit Games in Stochastic Network Models." In Combinatorial and Algorithmic Aspects of Networking: 4th Workshop, CAAN 2007, Halifax, Canada, August 14, 200\%, Revised Papers, Lecture Notes in Computer Science 4852, pp. 46-56. Berlin: Springer, 2007.

[Chung and Lu 03] F. R. K. Chung and L. Lu. "The Average Distance in a Random Graph with Given Expected Degrees." Internet Mathematics 1:1 (2003), 91-114.

[Chung and Lu 04] F. R. K. Chung and L. Lu. Complex Graphs and Networks. Providence, RI: American Mathematical Society, 2004.

[Cooper et al. 05] C. Cooper, R. Klasing, and M. Zito. "Lower Bounds and Algorithms for Dominating Sets in Web Graphs." Internet Mathematics 2:3 (2005), 275-300.

[Fomin and Thilikos 08] F. V. Fomin and D. Thilikos. "An Annotated Bibliography on Guaranteed Graph Searching." Theoretical Computer Science 399 (2008), 236245 .

[Hahn 07] G. Hahn. "Cops, Robbers and Graphs." Tatra Mountain Mathematical Publications 36 (2007), 163-176.

[Janson et al. 00] S. Janson, T. Łuczak, and A. Ruciński. Random Graphs. New York: Wiley, 2000.

[Łuczak and Prałat 09] T. Łuczak and P. Prałat. "Chasing Robbers on Random Graphs: Zigzag Theorem." Preprint, 2009.

[Neufeld and Nowakowski 98] S. Neufeld and R. Nowakowski. "A Game of Cops and Robbers Played on Products of Graphs." Discrete Math. 186 (1998), 253-268.

[Nowakowski and Winkler 83] R. Nowakowski and P. Winkler. "Vertex to Vertex Pursuit in a Graph." Discrete Mathematics 43 (1983), 230-239.

[Prałat 09] P. Prałat. "When Does a Random Graph Have Constant Cop Number?" Preprint, 2009.

[Quilliot 78] A. Quilliot. "Jeux et pointes fixes sur les graphes." PhD Dissertation, Université de Paris VI, 1978.

[Wieland and Godbole 01] B. Wieland and A. P. Godbole. "On the Domination Number of a Random Graph." The Electronic Journal of Combinatorics 8 (2001), \#R37. 
Anthony Bonato, Department of Mathematics, Ryerson University, 350 Victoria St., Toronto, ON M5B 2K3, Canada (abonato@ryerson.ca)

Paweł Prałat, Department of Mathematics and Statistics, Dalhousie University, Chase Building, Halifax, NS B3H 3J5, Canada (pralat@mathstat.dal.ca)

Changping Wang, Department of Mathematics, Ryerson University, 350 Victoria St., Toronto, ON M5B 2K3, Canada (cpwang@ryerson.ca)

Received November 22, 2007; accepted March 10, 2009. 